\title{
Organizational Diagnosis and Organizational Development Model: Integration of Psychoanalytic Determinants
}

\author{
Steven H. Appelbaum \\ Department of Management, John Molson School of Business, Concordia University, Montreal, Canada
}

Received March 19, 2020; Revised April 22, 2020; Accepted May 13, 2020

Copyright $\odot 2020$ by authors, all rights reserved. Authors agree that this article remains permanently open access under the terms of the Creative Commons Attribution License 4.0 International License

\begin{abstract}
This article reviews historical and current research focusing upon the failures and inadequacies associated with management and organizational development efforts, the role of the consultant in diagnosing organizational dysfunction. It further proposes a psychoanalytic model to serve as an organizational behavior blueprint. The model developed and processes identified are an intensive, prolonged technique to understand and identify the behavioral systems that trigger conflicts within the organization with significance attached to conflict and repression from early stages in the growth of an organization and its human resources-its people. The model synthesizes the research of psychoanalytic theory and organizational development (OD) processes in examining the components and effects of pain, affection, aggression, dependency and ego-ideal as interrelated forces. The impact of some of the historical founders of OD as well as Harry Levinson's psychoanalytic theory contribution will also be incorporated in this article. Examining relationships between intervention levels and component parts is the primary step to be taken in diagnosing, developing and changing the organization and environmental domain to an organic, reality-centered entity. The intra and interrelationships between the four intervention levels (individual, group, organization and environment) and the 30 variables uniquely, presents an overwhelming task for the organizational development consultant to diagnose, describe and hopefully change as the combination of psychoanalytic elements and organizational processes are complex and enigmatic at the individual/group levels with a multiplier effect illuminating the organizational and environment domains. The study of organizational diagnosis models is paramount for OD practitioners, because diagnostic models help to reveal key organizational gaps, and critical areas to focus. Moreover, this analysis emphasized the role of the OD practitioner as an advisor, helping clients identify the most
\end{abstract}

appropriate diagnostic model -given the intervention objectives, and the organizational culture, and using feedback mechanisms to channel results in a non-threatening mode to later focus targeted OD interventions for the improvement of organizational effectiveness. The development of a conceptual model intended to diagnose the individual and total elements of the system is the primary step to be taken in this change effort.

Keywords Organizational Diagnosis, Organizational Development, Psychoanalytic Theory, Intervention Levels, Management Development

\section{Background and History}

The conceptualization, development and commitment of organizations and their administrators to human resource programs are a tenuous, enigmatic process and event. Optimistically, organizations must assume that in the current complex high-technology world effective management will make the difference between successful and unsuccessful organizations (Pearse, 1974). This article will utilize a number of original and classic references from the origins of organizational diagnosis and development to be able to envision the path of these processes to the current time where psychoanalytic theory and models are still employed and deemed effective. Human resource development programs often fail for varied reasons and rationalizations, some of which are outside the domain of administrators. A philosophical commitment, or lack of one is attributable to aborted efforts and ruptured expectations. This article will attempt to identify those failures and inadequacies associated with management and organizational development (OD) efforts, the role of a 
consultant (interventionist), the introduction of a model of organizational diagnosis and organizational development and relationships between the individual, group, organization and environment. The utilization of a model is significant to this undertaking as the use of behavioral models to seek improvement in the fit between organizational units and their sectors of the environment is a required application (Lawrence and Lorsch, 1969). Lippitt (1973) endorses model building for administrators since modeling is a way to enhance one's awareness and insight into these complexities by improving the way that one perceives or sees the change situation. Managers need to develop new techniques knowing they live in a turbulent environment and constantly changing? On the contrary, if they can't develop new techniques and be involved in the process they will fail in their management and organizational development. They may wish to validate the model by testing out some scenarios for which they have some expectations of the outcome (McKenney and Keen, 1974). More than anything, the manager needs to learn how to tell a good model from a bad one. For the manager, a good model is one that they can, by testing their own scenarios, make sense of (Pearse, 1974). People tend to reject what they do not understand. The manager carries responsibility for outcomes. We should not be surprised if they prefer a simple analysis that they can grasp (Little 1970). This occurs more with those who are accustomed to utilizing hard skills. It appears to be clear that diagnosis, intervention and development are processes that must be realistically nurtured within organizations as they incubate, mature and perpetuate themselves.

\section{Problems with Adapting to Management Development and Organizational Development}

Organizational and management development efforts basically fail because top management only intellectualizes a commitment, but does not internalize the gut-level ramifications of the project. Programs are often formalized and force-fed to candidates not actually involved in the decision-making process. The projects are limited by time is a reality, and not only 'often' limited by time, but based upon popular, faddish techniques associated with manipulated outcomes and are unreliable and unpredictable since the formal culture may consist of idealized statements of what the values, beliefs and behaviors of members should be. The informal culture may consist of these as they really are (Margulies and Wallace, 1973). Organizations do not have a social responsibility to develop a "brave new world" for their valued human resources. Formal culture is all habits, myths, rites, mores and folkways and it is an organizational routine shared by all the actors. The problem in this case is not the presence of a formal culture or not one, but with individual micro-cultures. There is the organizational culture and subcultures specific to each actor, each of them has its own objectives. This is what can make organizational development fail. Cultures are often felt and not clearly seen which makes reading them enigmatically.

Individuals earmarked and designated for development should not be required to participate in those programs unless the program has intrinsic worth and positive reinforcement for them which is incorporated within the design. These individuals must intrinsically feel they possess the talent, judgment and drive to successfully participate in and complete programs designed "for their growth and benefit" which is a highly personalized decision often handled ambivalently. This can occur satisfactorily if the individual is overtly cognizant of a top management commitment and is permitted to realistically apply development outcomes, theories and experiences to their current responsibilities within the uncertainties of the organizational environment. Effectiveness occurs when developmental projects try to objectively identify "real" administrative and behavioral problems facing "real" individuals who possess certain capabilities and inadequacies with a reality-centered environment. Individual growth is identified and demonstrated when "trainees" begin to make rational decisions in complex organizational situations. The realization of this outcome is based upon a competent and objective appraisal of performance, potential and development needs by an external or internal consultant incorporating a human resource blueprint intended to move the individual from point $\mathrm{A}$ to point $\mathrm{B}$. When managers evaluate the relative worth of training courses they have taken, they say courses on interpersonal relationship skills are about twice as beneficial as courses on improved personal effectiveness (Pearse, 1974). Optimum effectiveness is more realistic when development efforts are directed toward the total organizational climate and encourages application and testing of program theory and experiences via encouragement and reward to those seeking additional responsibilities while utilizing new behaviors. Classical and foundation considered behavioral science- based motivational theories such as McGregor's Theory $\mathrm{X}$ and Theory Y (1957), Maslow's need hierarchy (1943), Herzberg's satisfiers-motivators (1968), and Blake and Mouton's Managerial Grid (1964) have historically been used in management to explain some of this. About one out of five managers who had been exposed to such theories found they were able to apply them on the job with considerable effectiveness (Pearse, 1974).

\section{Management Development and Organizational Development Foundations}

Management development cannot succeed and exist as 
an entity within its own vacuum. It must be the major component within the broader sphere of organizational development, a long-range effort and commitment to improving the problem-solving capability of an organization while sensitizing it to anticipate, cope with and manage change. The development of a model, serving as an ideational, human resource blueprint is often conceptualized by a consultant, emphasizing relationships and outcomes which should exist between management and organizational development. A synthesis of both efforts is the essential focal point as described by Lippitt (1973):

Basically, a model is a symbolic representation of the various aspects of a complex event or situation, and their interrelationships... The true value of a model lies in the fact that it is an abstraction of reality that can be useful for analytical purposes . . . through the use of models, they can predict performance under predetermined conditions or evaluate the consequences of various alternatives before committing themselves to a particular change plan or course of action.

When management development is intellectualized and internalized by top administrators as being necessary for successful organizational development, the level of trust and support among organizational members (French, 1972) is intensified. For further insights see Smith (2019) and Bunjak (2019). In addition, individual feelings of self-worth are escalated. Authority and power relationships must also be diagnosed and identified while win-lose strategies between people and groups, while realistic and appropriate in some situations, are not optimal in the long run to the solution of most organizational problems (French, 1972) and to developmental efforts. The consultant assumes an important role in this context as he must adapt the organization to changes in the internal/external environment (Appelbaum, 1973) and present an important question to key administrative leadership anticipating a return on development investment: Does the formal reward structure set up a situation in which destructive competitiveness both between individuals and between organizational sub-units become a necessary condition for reward (Margulies and Wallace, 1973)? Conflict, a result of competition, must be realistically neutralized and hopefully reduced because it is essential for the individual experiencing a developmental program of change to become aware of his/her personal feelings, impact upon the department and group and attitude toward the organization encouraging his/her development. (Appelbaum, 1977). Effectiveness is realized when organizational development emphasizes trust, support and reward as components to share interdependency rather than isolationism. Organizations encourage structure, reward/punishment and control when efforts to develop administrators with lower than required education and experiences are being "pushed" to achieve higher status and organizational levels than they are technically and/or psychologically capable of reaching, ultimately intensifying anxiety, fear and failure. Unrealistic expectations and failure result in a shattered ego-ideal, low self-image, self-directed aggression, guilt and finally depression. It is not difficult to see how development efforts become resisted and obliterated.

\section{The Consultants Approach to Organizational Development}

John Kotter introduced the "Kotter's 8 Step Change Model" (Kotter, 1996) to improve an organization's ability to change and to increase its chances of success. By following this step plan organizations can avoid failure and become adept at implementing change. The model is as follows:

\subsection{What Is Kotter's 8 Step Change Model?}

Research carried out by the change and leadership guru has proved that major change efforts unfortunately do not always have the desired outcome. He found out that there is only a $30 \%$ chance of organizational change success. This is why organizations implement changes unsuccessfully and fail to achieve the intended result. Kotter introduced the "Kotter's 8 Step Change Model" to improve an organization's ability to change and to increase its chances of success. By following this step plan organizations can avoid failure and become adept at implementing change. As a result, organizations no longer need to adjust the changes and they will increase their chances of success.

\subsection{Create a Sense of Urgency}

This first step of Kotter's 8 Step Change Model is the most important step. By making employees aware of the need and urgency for change, support will be created. This requires an open, honest and convincing dialogue. This convinces employees of the importance of taking action. This could be accomplished by talking with them about potential threats or by discussing possible solutions.

\subsection{Create a Guiding Coalition}

It is a good idea to establish a project team that can occupy itself with the changes the organization wants to implement. This group manages all efforts and encourages the employees to cooperate and take a constructive approach. Preferably, this coalition is made up of employees working in different jobs and positions so that all employees can rely on the group and identify themselves with the team members. Because of the open character, the groups can also function as a sounding board, which enables open communication.

\subsection{Create a Vision for Change}

Formulating a clear vision can help everyone understand 
what the organization is trying to achieve within the agreed time frame. It makes changes more concrete and creates support to implement them. The ideas of employees can be incorporated in the vision, so that they will accept the vision faster. Linking the adopted vision to strategies will help employees to achieve their goals.

\subsection{Communicate the Vision}

The most important objective of step 4 of Kotter's 8 Step Change Model is to create support and acceptance among the employees. This can only be achieved by talking about the new vision with the employees at every chance you get and by taking their opinions, concerns and anxieties seriously. The new vision must be fully adopted across the entire organization.

\subsection{Remove Obstacles}

Before a change is accepted at all levels, it is crucial to change or, if necessary, remove obstacles that could undermine the vision. By entering into dialogue with all employees, it will become clear who is resisting the change. To encourage acceptance of the vision by the employees, it helps when their ideas are incorporated and implemented in the change process.

\subsection{Create Short-Term Wins}

Nothing motivates more than success. Create short-term goals so that the employees have a clear idea of what is going on. When the goals have been met, the employees will be motivated to fine-tune and expand the change. By acknowledging and rewarding employees who are closely involved in the change process, it will be clear across the board that the company is changing course.

\subsection{Consolidate Improvements}

According to Kotter, many change trajectories fail because victory is declared too early. However, change is a slow-going process and it must be driven into the overall corporate culture. Quick wins are only the beginning of long-term change. An organization, therefore, needs to keep looking for improvements. Only after multiple successes have been achieved, it can be established that the change is paying off.

\subsection{Anchor the Changes}

The last step of Kotter's 8 Step Change Model. A change will only become part of the corporate culture when it has become a part of the core of the organization. Change does not come about by itself. Values and standards must agree with the new vision and the employees' behaviour must provide a seamless match. Employees must continue to support the change. Regular evaluation and discussions about progress help consolidate the change.

The most general lesson to be learned from the more successful cases is that the change process goes through a series of phases that, in total, usually require a considerable length of time. Skipping steps create only the illusion of speed and never produce a satisfying result. A second very general lesson is that critical mistakes in any of the phases can have a devastating impact, slowing momentum and negating hard-won gains. Perhaps because we have relatively little experience in renewing organizations, even very capable people often make at least one big error.

Many organizations, in an attempt to adapt to the constant evolutions of their environment, are adopting cultures of a learning or agile organization. Whether or not an organization tries to constantly evolve, successfully implementing changes can be a major determinant of its short- and long-term success. Considering that "research suggests that failed organizational change initiatives range from one-third to as high as $80 \%$ of attempted change efforts" (Appelbaum, et. al, 2012). Although Kotter's model of change management lacks rigorous fundaments, it became an instantaneous success at the time it was advocated and it remains a key reference in the field of change management. In 1997, Leading Change (Kotter, 1996) became a business bestseller. It subsequently became the best-selling book ever of its kind. Hundreds of researchers refer to one or other of Kotter's publications on change management. This book has been cited over 5,000 times in Google Scholar.

The general lack of empirical fundaments to most change management theory (Todnem, 2005) probably is not alien to this success. "Theories and approaches to change management currently available to academics and practitioners are often contradictory, mostly lacking empirical evidence and supported by unchallenged hypotheses concerning the nature of contemporary organizational change management" (Todnem, 2005). The success of the theory and at the same time the lack of research and rigorous investigation is quite counterintuitive in the world of empirical research that has been the underpinning of accepted $\mathrm{OB}$ and $\mathrm{OD}$ references and classics. (Appelbaum, et. al, 2012).

The development of a model intended to affect the organization and its efforts and commitment to manage change and human resources is the initial step taken by the organization experiencing pain, dysfunctional operations and inhibited growth. For further insight see Parveen and Adeinat (2019). Behavioral expertise must be provided to critically diagnose incongruencies and recommend alternatives-hence the entry of the consultant in the dual role of buffer/catalyst and central figure around which the organization, management philosophy and motivational systems evolve. This interrelationship is presented in Figure 1 (designed by Appelbaum). While this framework is descriptively viable, there are no widely accepted comprehensive diagnostic procedures for assessing and 
evaluating organizations (Levinson, 1972).

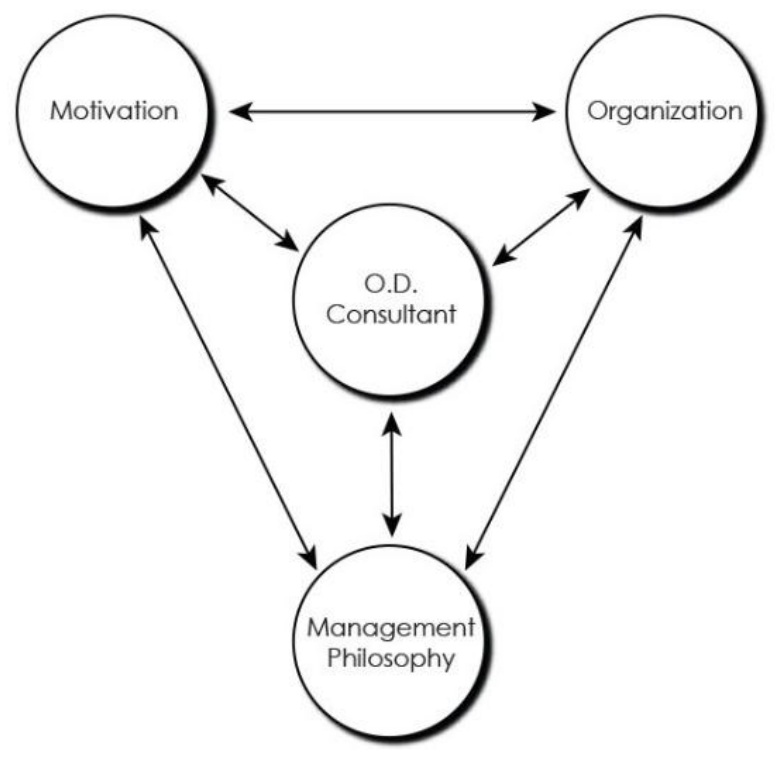

Figure 1. A consultative O. D. model

An external consultant yields obvious advantages but an internal figure is overt, possesses professional power, is visible and felt and has far more potential than most organizations realize (Margulies and Wallace, 1973).For further insight see Vosoughi (2019), To be effective, the consultant should not occupy a formal organizational status position which increases vulnerability to conflict, manipulation and compromise. Interestingly, a significant part of the external consultant's strategy is, from the very beginning, explicit planning for the development of internal change agents (Margulies and Wallace, 1973). The consultant, even though internally situated must direct the key administration to clarify the role and responsibility relationships of individuals and groups while recommending those programs intended to strengthen leadership and improve managerial skills in problem-solving and group goal-setting (Appelbaum, 1973).

The interactive elements of the proposed model are integrated into the organizational supersystem as this approach illuminates the behavioral components associated with open, complex systems such as thoughts, feeling and perceptions which become both inputs and outputs of the O.D. effort. What is actually being proposed is a model for administrators to refer to and utilize in describing behavior within their organization. The model should be psychoanalytic since this undertaking is an intensive and prolonged technique to explore organizational motivation systems with importance attached to conflict and repression from early stages in the growth of the organization. It appears to be important to bring to the surface any, and hopefully most, repressed conflicts so that they may be resolved within a reality-centered climate. This is why the O.D. consultant must be strategically located at the hub of conflicting forces within the organization, and within the proposed model as well, so that a lucid diagnosis can be made. Diagnosis is a primary phase of a consulting relationship since a diagnostic method should also be a basis for training people for organizational consultation, and for fostering and formalizing a professional consultation role (Levinson, 1972).

\section{Organizational Diagnosis}

Organizational diagnosis is a creative method for getting to know an organization at all levels- from the surface levels to the deepest hidden parts that aren't visible to the eye. Performing organizational diagnosis is not so far off from a doctor trying to diagnose their patients. Some doctors diagnose differently by focusing on nutrition, food, and natural remedies, whereas others diagnose by using pharmaceutical medications, or even by trying a remedy, seeing whether it has positive effects, and then trying something new. This is very similar to what we have learned to do in the business sense to organizations. Different diagnostic models can be used in different situations depending on the wants, needs, and goals of our clients (patients). (Furgoch, 2016). Generally speaking, these models would be used by human resources or organizational development and change practitioners who either work internally in an organization, or have been hired as a contracted consultant to help ascertain the root causes of issues an organization is facing and to provide recommendations to improve them. In either case, the diagnostic models provide a template or tool to break down the organization into components to understand it more in depth as well as to better visualize how all of the parts work together. From performing the diagnosis alone, it is often possible to begin to pick out issues that are present within an organization. (Furgoch, 2016). For further insights on diagnosis see Noolan (2006).

OD diagnostic models draw from the action research practice, where clients are involved and take an active role in the many stages of the diagnosis process, including the definition of objectives, stakeholders, activities, and timeframe to gather and analyze data. The key player concerned by action research is above all the managers, the client is involved in the organizational development process through the participatory approach. The role of the OD practitioner is paramount to guide clients in the organizational assessment process, including the selection of the diagnostic model, the definition of activities, the feedback of results and the implementation of following actions to improve organizational effectiveness and individual and organizational development. For further insights see Lundberg, (2008).

The legitimization of this diagnostic process begins with a thorough, insightful examination of the history of the 
organization, the value systems, human resources, patterns of crisis management, long-term programs, recruitment and socialization of personnel, personality types, manipulative efforts, internal and external image of the organization under study, adaptation and coping with change, developmental stages of growth and any revitalization efforts.(For further insights see Bushe and Marshak,2009). The purpose of this perusal is to gain understanding, interpret primary data and develop tentative inferences since the diagnostic process refers to the early stages of the relationship between consultant and organization, a series of interactions and transactions which hopefully will lead to constructive organizational change (Levinson, 1972). The diagnostic phase of an organizational study must be a mutually beneficial experience since a consulting relationship, like a therapeutic or teaching relationship should be an alliance of both parties to discover and resolve problems (Levinson, 1972). The formalization of this phase should be in the form of a written process report (case study) to be presented to top administration, the Chief Executive Officer (CEO), by the consultant highlighting clearly stated strengths, weaknesses, support needed and prognosticated capacity for change.

This process and intervention phase of a diagnostic/developmental effort gives early relief from some painful organizational symptoms since change efforts are symbolically perceived as loss which can be interpreted as personalized by the CEO. Unless an open climate is developed, permitting ventilation of fears and anxiety, a therapeutic catharsis will virtually be non-existent thereby retarding change and developmental efforts. The consultant actually assumes the role of an organizational therapist to enlighten and yield insightful understanding to top administration. This event enables the organizational depression to surface which is causing pain, ineffectiveness and dysfunction. The energization of this process reduces the disparity between perceptions, expectations and reality. This congruence is a positive indicator of the prognosis of organizational behavior and health. As organizations or parts of organizations become better tailored to their tasks, individuals can increasingly select their work roles on the basis of individual preference, secure in the knowledge that the associated organizational disciplines will be task-based rather than arbitrary (Lawrence and Lorsch, 1969).

\section{A Psychoanalytic Approach and a Diagnostic Model}

Harry Levinson was a clinical psychologist by training whose considerable body of writing and practice helped to pioneer the field of organizational consulting psychology. His books and articles have been historically popular both with managers and with psychologists who would consult with organizations. The particular model that Levinson has persistently espoused throughout his professional career is psychoanalysis. How often do companies call in organizational diagnosis (OD) experts and ask for team building, conflict management, or values alignment with absolutely no interest in, or willingness to pay for, preliminary assessment work? It is not that such treatments are unnecessary or cannot in some circumstances do some good - most companies probably can use interventions of this sort. For further insights see Stern, Cifu, and Altkorn, (2010). However, as Harry Levinson has taught us, without competent assessment there is no assurance that these treatments address what ails the organization or are likely to result in long-lasting change. (Lowman, p.20-21). Levinson has also taught us that there is no substitute for judgment, clinical and otherwise, in integrating across multiple sources of complex data to draw inferences and conclusions (see Levinson, 2002a, 2002b, 2002c).

How does the psychologist-manager make use of organizational diagnosis and of Levinson's models? He suggests we tie the results of the assessment to the contemplated actions. Before acting, psychologist-managers need a plan based on an understanding of what an organization needs and how the planned changes relate to those needs. The intervention plan should be based not just on the business or organizational needs but also on an understanding of the psychological aspects of the situations at hand. (Lowman, p.27). Psychologist-managers attempt interventions in their organizations that have a known track record. There is now more substantial empirical research literature on what works and what does not in organizational consultation (see, e.g., Halfhill,Huff, Johnson, Ballentine, \& Beyerlein, 2002). Psychologist-managers understanding and practicing the relevant literature need to make use of it in their actions. There is so much more that could be said about organizational diagnosis and the special contributions of Harry Levinson. The professional challenge of learning how to conduct organizational assessments in a valid and reliable way — what works and what does not-is not over; it is really just getting started. However, thanks to the work of Levinson, we-all of us who aspire to conduct organizational assessments in a way that creates meaning, relieves pain, and helps to promote organizational well-being. (Lowman, p.27).

Any model is valuable when it improves our understanding of obscure behavioral characteristics more than would be possible by observing the real system. A model can more quickly provide knowledge of conditions not observable in real life (Lippitt, 1973). Models have developed into blueprints for behavioral scientists and consultants to emulate in organizational intervention efforts but since the 1950's it has been possible to construct in a laboratory realistic models of social systems. These models are simplifications of actual social systems (Lippitt, 1973). Models utilized for diagnostic purposes possess these advantages: (1) models allow experimentation 
without risk; (2) models are good predictors of system behavior and performance; (3) the relative significance of various factors can be determined; and finally, models demonstrate the interrelationships of multiple factors (Lippitt, 1973) to be considered, diagnosed and examined by the organization and consultant.

The consultant (internal or external) experiences success only when pain and its symptoms are illuminated in terms of the entire life process of the organization prior to developing remedies for growth, independence, innovation, etc. The psychoanalytic model is a viable and valid diagnostic procedure as traditional psychodynamic theories of change, drawn largely from psychoanalytic thought, most typically present the problem of change as a largely internal affair (Margulies and Wallace, 1973). If we are to envision the individual within the development-change process as a microcosm of the organization seeking changes and new behaviors, growth is an intended outcome since organizational growth cycles are closely akin to the growth cycles of human beings. Organizations certainly experience periods of conception, birth, the survival stage, childhood, adolescence, and on through adulthood (Spencer, 1974). Therefore, development must be perceived as a maturing process as maturity is a constantly evolving process marked by progress, fixations and regressions (Spencer, 1974). This classical observation is still valid in 2020 .
Life within organizations and human systems must be perceived as being congruent because only when senior management understands the relationship of organizational maturity to long-term productivity and actively seek to positively affect the growth stages, can we hope to avoid the unnecessary and self-defeating behaviors which contribute to the failure of four out of five businesses (Spencer, 1974) and most well-intended organizational development efforts. For further insight sees Wong, et.al. (2019) and Vilkinas, et.al. (2019). Levinson (1972) summarizes this concept by stressing to understand an organization as a living entity, it will be important to see organizational purpose as being akin to the issue of identity for the individual. An organizational purpose implies a concept of the organization, a self-image if you will. Development requires the integration of individual, group and organization, each presented earlier in the Model, for the purpose of maintaining identity and perpetuation of the organizations themselves. The psychoanalytic model of organizational diagnosis proposed in Figure 2 (designed by Appelbaum) focuses upon the individual, group and organization by examining the components of pain, affection, aggression, dependency and ego-ideal as interrelated factors. Each one of these critical factors will be covered in terms of the broader universe that is affected by each one.

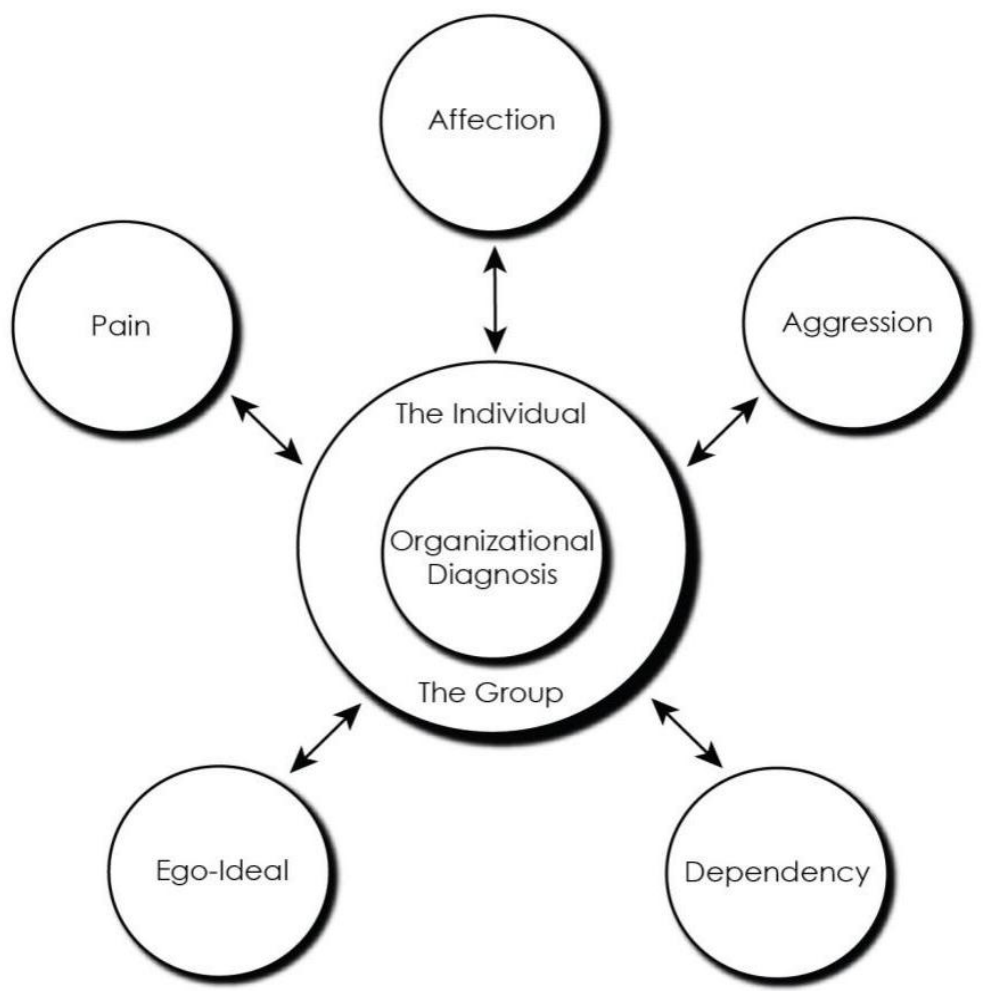

Figure 2. Psychoanalytic model of organizational diagnosis 


\section{Pain}

There are two reasons why one person comes to another for help; either he has some kind of pain (suffering, discomfort, problem) or he is causing someone else to have such pain who is in turn compelling him to do something about that problem (Levinson, 1972).

The consultative relationship is essential in diagnosing pain felt both organizationally and individually for if there is no pain felt by either party, no problem actually exists requiring intervention or action. But, if the pain is apparent, then the initial questions to ask are:

Where is the pain?

How long has the pain existed? Has it always been this way?

When did changes occur?

Are there new forces present to account for pain felt? Is the pain acute? Is it chronic?

Is pain being denied when actually felt?

Pain within organizational members and the system causes anxiety which may be difficult to diagnose and manage since the following chain is employed by organizations seeking to deny or repress overt or covert pain:

ANXIETY CONFORMITY CONTROL ANXIETY

This philosophy and technique often intensify the pain as individuals experience heightened anxiety when conformity and control are prerequisites to pain reduction. Integrating affection and its symbols into the organizational framework while balancing or neutralizing aggression and dependency is essential in pain reduction. Unresolved attempts to reduce pain emerging from the anxiety, conformity control syndrome leads to a depressed state inhibiting development and growth efforts.

\section{Affection}

Affection is often symbolized by organizations in the form of compensation, achievement, recognition and other tangible elements for individuals. There appears to be an implied contract at all times between the individual and organization in the form of an inducement's contributions agreement. Compensation and other elements are perceived and utilized as organizational affection symbols, as a trade-off for individuals yielding contributions in the form of loyalty, efficiency and commitment. Contributions, however, must be balanced by inducements proposed by March and Simon over a half-century ago who were the earliest theorists in $\mathrm{OB}$ such as income, status and professionalism given by the organization:

Each participant and each group of participants receives from the organization inducements in return for which he makes to the organization's contributions.
Each participant will continue his participation in an organization only so long as the inducements offered him are as great or greater (measured in terms of his values and in terms of the alternatives open to him) than the contributions he is asked to make (March and Simon, 1958).

The consultants must determine whether affection or its symbolism is rewarded, understood, displaced, contained or redirected. This diagnostic phase of a development effort is necessary since organizations have the power to manipulate affection often causing dissonance within the individual. This state of uncertainty illuminates' anxieties, control and finally compliance. While organizations follow their own models of affection-rewards, this situation is characteristic of paternalistic systems possessing a benevolent philosophy in which responsibility is felt and assumed for the employee from cradle to grave. A dependency relationship is thus developed where growth and intrinsic development are thwarted. Some fallout from this is often expressed in aggression.

\section{Aggression}

Organizations are developed and driven to accomplish and achieve. Individuals possess drives needed to satisfy objectives, but these drives are often displaced, misdirected or redirected in which the target and goal are aborted, intensifying frustration and failure. Individual drives that are necessary for organizational goal accomplishment can be funneled into tasks, or displaced, or contained and held onto. However, unless channels are available for displacement or ventilation, drives can be redirected and turned destructively against the individual attempting to understand and cope with them. The internalization of frustrating drives manifests itself within the individual in the form of anger and hostile withdrawal from others. The consultant must balance this uncertain state against developmental programming because anger is dysfunctional.

Anger manifests itself more subtly in the displacement of hostility onto others (Levinson, 1970) causing the sub-optimization of development plans. However, anger which is overcontrolled reflects itself in tension, irritability and pain (Levinson, 1970), equally disabling to an organization. Development programs intensify anxiety which should be understood by the individual concerning this uncomfortable, heightened state brought about by new behavioral demands being made upon him/her. Dependency needs can become more acute if the new job is not adequately structured so that an individual can continue to have ready access to his superiors, or if the job is not clearly defined, or if the individual has not had enough experience to cope with it (Levinson, 1970). Any and all of these situations cause frustration, then anger and finally aggression which seeks outlets to balance understanding 
and cognizance. Development programs must focus upon this syndrome for realistic outcomes if success is to be realized. It is essential for organizations to recognize and consultants to diagnose the fact that sometimes people have so much difficulty in dealing with their unconscious feelings of anger that the only way they can get rid of them is to hurt themselves in some manner (Levinson, 1970). But, when organizational ventilation systems do not permit the displacement of aggression, individuals not only hurt themselves but also sabotage the organization via low productivity, absenteeism and distorted communications. When organizations seek to control the individual, repressed feelings- anger-aggressions become destructive when the individual internalizes those drives leaving him/her in a depressed, ineffective and unproductive state, where dual objectives become out of reach. An outcome of this state is often and unfortunately dependency.

\section{Dependency}

Argyris (1964) hypothesized that there is a lack of congruency between the needs of individuals aspiring for psychological success and the demands of the formal organization. He further stated that the results of this disturbance are frustration, failure, short-time perspective and conflict. The effect of this incongruency is low selfesteem, but, psychological success is therefore hypothesized to be the mechanism for increasing self-esteem. Argyris (1960) in his classic study hypothesized that individuals:

Tend to develop from a state of dependence upon others as infants to a state of relative independence as adults. Relative independence is the ability to stand on one's own two feet and simultaneously to acknowledge healthy dependencies.

It is evident that a dilemma exists between the need for the individual to maintain the independence and a corresponding organizational need to keep him/her in a dependent position due to needs of control, predictability and conformity. The result is an irreconcilable conflict. Dependent positions are uncomfortable and incongruous to successful development efforts by organizations for adult managers. Dependency conditions and feelings are experienced at infancy recur in later life in organizations where the same infantile patterns may prevail at adult levels. It is common and normal for individuals to feel uncomfortable when they are dependent on someone else. Some people cannot tolerate accepting favors; others find it difficult to turn to someone else for help, no matter how badly they need it (Levinson, 1970). Therefore, it is essential for the consultant to determine how dependency is handled and supported prior to commencing development programs. Leadership support is an ingredient for program effectiveness as many administrators, due to personality traits, cannot learn (depend) on anyone because of a distorted self-image and an inadequate foundation.

If a man feels he cannot do anything about these forces (inadequacy) he stops trying and becomes apathetic... becomes dependent on someone else, and being dependent feels childlike ... to be master of himself and the forces that affect him, a man must continue to grow psycho- logically (Levinson, 1970).

This situation is often reflected in pseudo-participatory management attempts to develop those individuals who have not been diagnosed and requested to verbalize their expectations and development outcomes. Organizational structures and systems foster dependency relationships by denying personnel access to information and power sources. Paternalism poses a similar problem by showering employees with promises of benefits and then making it clear that gratitude is expected by submission. These behaviors affect the individual and his perception of his/her competence and self-image which leads to the defense mechanism of infantilization. Developmental efforts and psychological success are therefore hypothesized to be the mechanism for increasing self-esteem (Argyris, 1964) which neutralizes dependency:

In order to experience psychological success, three requirements are essential. The individuals must value themselves and aspire to experience an increasing sense of competence. . . The second requirement is an organization that provides opportunities for work where the individual is able to define his immediate goals, define his own paths to these goals, relate these to the goals of the organization, evaluate his own effectiveness, and constantly increase the degree of the challenge at work.

\section{Ego-Ideal}

The ego-ideal as a component of the psychoanalytic model and relationship to development efforts poses a challenge to the consultant (internal or external) in their organizational diagnosis and audit of human resources. A basic dualism exists between the ego- ideal of the firm and individual since the ego-ideal and self-image are closely related. An organization's ego-ideal is what it thinks it should be; its self-image is what it thinks it is. The distance between the two is an index of self-esteem (Levinson, 1970). This is a consideration for developmental efforts because individuals whose self- esteem is easily threatened and/or low, are less likely to be rational about their efforts to satisfy their individual needs and less receptive to organizational development programs. The diagnosis of organizational objectives as being profitable, social responsibility or even organizational perpetuation is needed as a blueprint to balance an inquiry into individual needs. The integration of both efforts into a realistic model clearly distinguishes between lucid and/or distorted expectations. Generally distorted perceptions can be dealt 
with and handled by the consultant within a development program, but distorted expectations pose a different enigmatic problem:

If there is a wide gap between the image he projects and the person, he really is, emotional conflicts are inevitable. The same is true of a business organization: the greater the gap between what an organization pretends to be and what it really is, the more internal conflict there is likely to be. When public posture and reality divide, difficulties will arise (Levinson, 1970).

Frustration is a feeling and not a fact. Therefore, the O.D. consultant legitimizes their role by diagnosing first and later feeding back to those individuals involved within development programs' perceptions on how they would like to see themselves as contrasted with how they really do perceive themselves. When this differential is presented openly, distorted expectations and frustrations are illuminated, dealt with, and neutralized. The ego-ideal and development efforts crumble because if people cannot share an idea, they cannot be psychologically close to each other and cannot sustain a relationship (Levinson, 1970). If organizations are systems composed of interdependent components and interrelationships, goals (ideals) are foundations or models upon which effective efforts must rest.

\section{Conclusions: Putting It All Together--The Individual, Group, Organization and Environment}

The need for and objectives of organizational development has created the psychoanalytic model presented earlier in this undertaking. The focus of the approach is upon the internal climate of the organization in order to diagnose those forces and symptoms affecting successful change efforts. But an organizational diagnosis is incomplete unless the impact and effect of the individual upon the group, the organization and environment are studied in conjunction with an inverse proportional analysis of environmental impact upon the organization, group and individual as well. This diagnosis is significant for managers in the system as well as the consultant as their point of intervention is dependent upon this interface. The relationship and description of the integrative elements are presented in Figure 3 (designed by Appelbaum). It is important to note that the organizational development model cannot be complete without identifying the internal and external organizational culture which explains all the phenomena including negative emotions. Furthermore, the degree of importance of the emotional negative impact on the individual alone compared and contrasted with the level of impact when considering the group, and within the group where the emotional shock may be well endured by individuals. Finally, and a critical point in Figure 3, is to integrate into this model the technology into the organization's internal environment like the science (the method, the technique, the tool, etc.), because it has a direct influence on the motivation of human resources. This is one of the key challenges for the OD consultant and key leadership.

The O.D. consultant intervenes within a system at a point at which they diagnose felt pain and at a level that they objectively perceive their competencies to be most effective for optimum success. The point of intervention and level of consultant competency can focus upon the individual, the group, or the organization. The ramifications of accurate information follow the lifeline of future organizational survival and will depend on two areas: (1) successful human resource utilization and development, and (2) successful adaptation to a rapidly changing external environment (Pearce, 1974). Intervention at the individual level and developmental efforts is important considerations because living organisms have energy. They can act forcibly on their environment. They are not merely acted upon (Levinson, 1972). If intervention is to occur at individual or group levels, the psychoanalytic model and all considerations must be examined with regard to pain, affection, aggression, dependence, ego-ideal, and expectations. Distorted expectations retard intervention and developmental effectiveness. Groups within the organization are subject to similar experiences, development and expectations as individual members, therefore, an organization of any appreciable size must form groups or units for the performance of the different kinds of activities (tasks) that are required for the execution of its selected transactional strategy (Lawrence and Lorsch, 1969). 


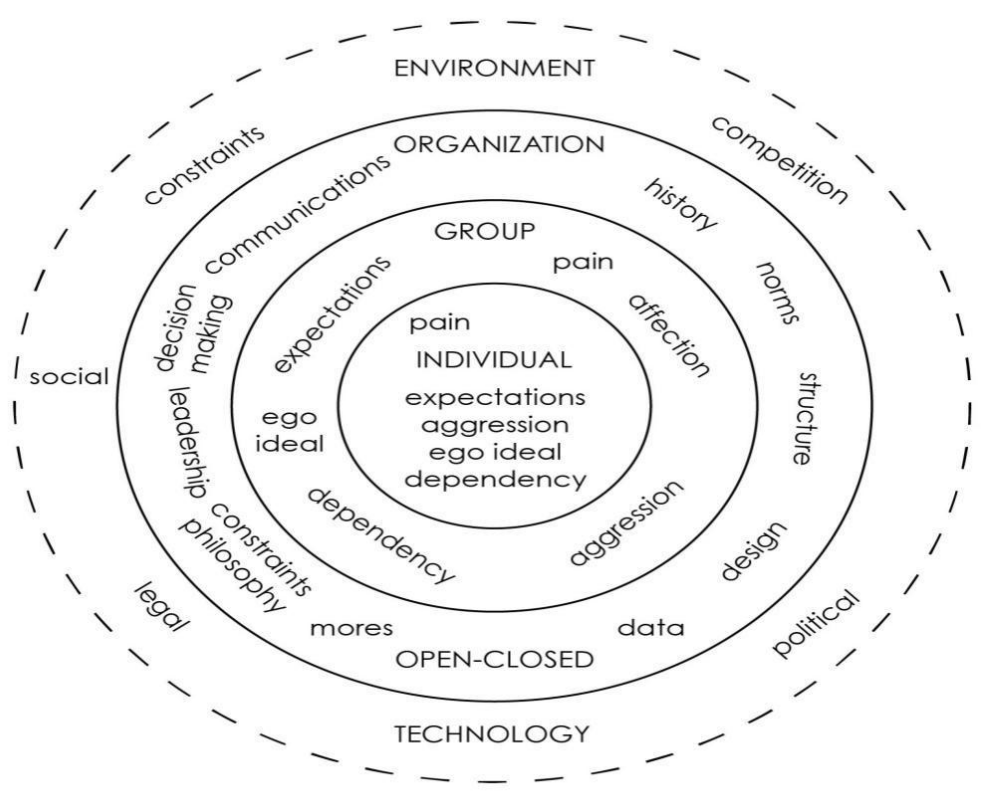

Figure 3. An integrative model of organizational diagnosis

Development efforts and actualization of these efforts can become displaced or diluted since groups, once formed, will evolve their own distinctive task-related characteristics, they will have different points of view that necessarily complicate the coordination process (Lawrence and Lorsch, 1969). Integrative efforts, on the part of the consultant, are essential to overcompensate for any sub-optimized developmental plans because organizations too, suffer insult and injury from their environments or conflict because of contradictory goals and purposes (Levinson, 1972).

When the consultant experiences dissonance in intervention at the individual or group levels, the organization and its relationship with the environment must be carefully examined for constraints and alternative change strategies. The sensitive relationship and interface between the individual organization and the environment is presented in Figure 4 (designed by Appelbaum), and is essential for total cognizance since Levinson states all of us must cope with the environment in which we live; from the air we breathe to the dust we become, we are a part of it and it is a part of us (Levinson, 1970).

Integration occurs because the organization becomes a device for mediating between the individual and his wider environment. It provides a setting that structures and channels his transactions with the environment (Lawrence and Lorsch, 1969). Integration is not a by-product of a consultative effort to interface those elements interdependent upon each other for perpetuation and growth/development because it is no mystery that organizations must carry on transactions with their environment simply to survive, and even more important to grow (Lawrence and Lorsch, 1969).

Therefore, a diagnosis focusing upon the organization and those aspects of the organization which must be explored in depth by the consultant in order to illuminate the intricate relationships affecting development and change efforts is essential. The organization and its twelve internal processes to be diagnosed and then fully examined are presented in Figure 5 (designed by Appelbaum).

The 12 processes yield a thorough internal perspective of the organization and potential points of intervention. This diagnosis cannot be effectively realized without a similar examination of the environment of the organization. These six factors to be considered are presented in Figure 6 (designed by Appelbaum).

The intra and interrelationships between the four intervention levels (individual, group, organization and environment) and the 30 variables just examined, presents an overwhelming task for the organizational development consultant to diagnose, describe and hopefully change as the combination of psychoanalytic elements and organizational processes are complex and enigmatic at the individual/group levels with a multiplier effect illuminating the organizational/environment domains. For further analysis see Armenakis and Harris (2009).

The study of organizational diagnosis models is paramount for OD practitioners, because diagnostic models help to reveal key organizational gaps, and critical areas to focus. Moreover, this analysis emphasized the role of the OD practitioner as an advisor, helping clients identify the most appropriate diagnostic model -given the intervention objectives, and the organizational culture, and using feedback mechanisms to channel results in a non-threatening mode to later focus targeted OD interventions for the improvement of organizational effectiveness. The development of a model intended to diagnose the individual and total elements of the system are the primary step to be taken in this change effort. 


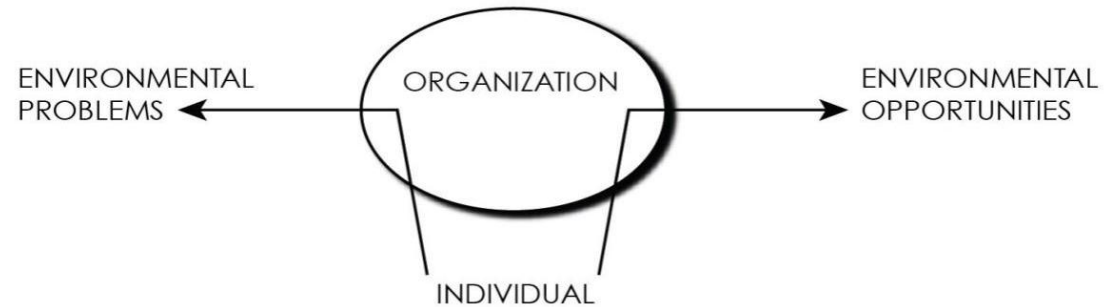

Figure 4. Environmental interface

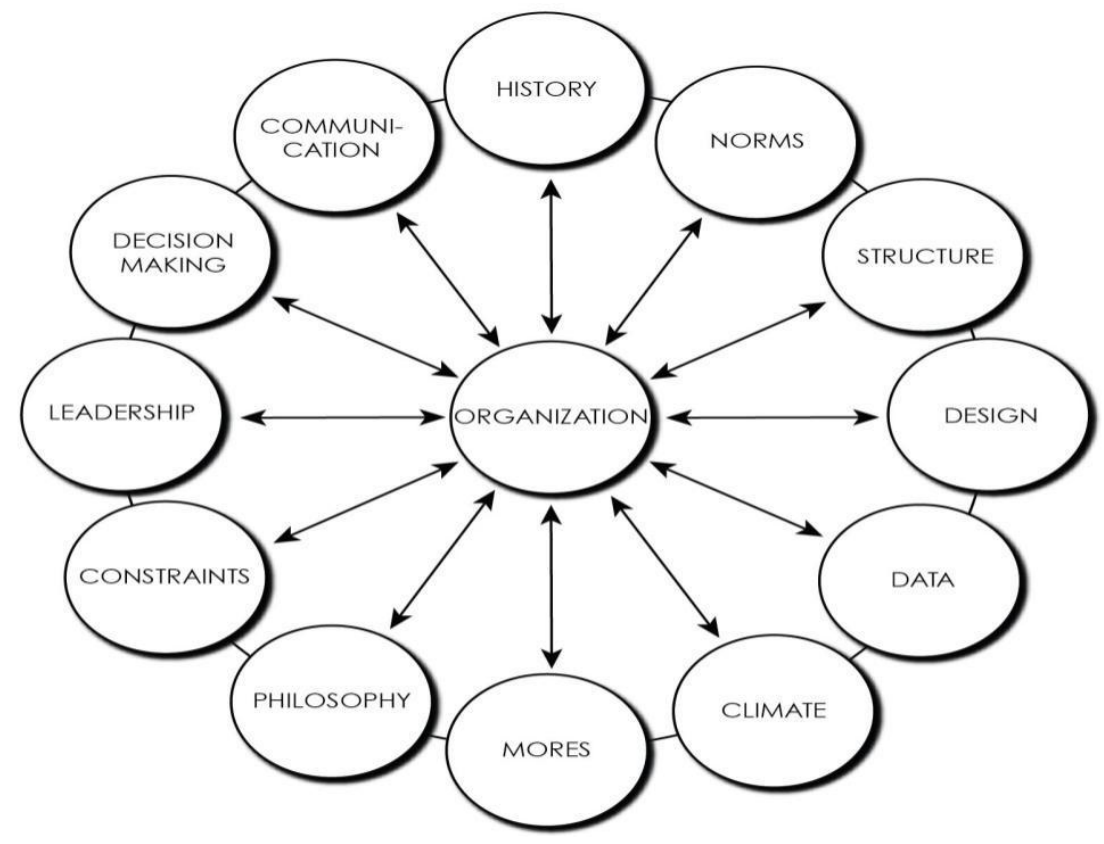

Figure 5. A model of internal organizational processes

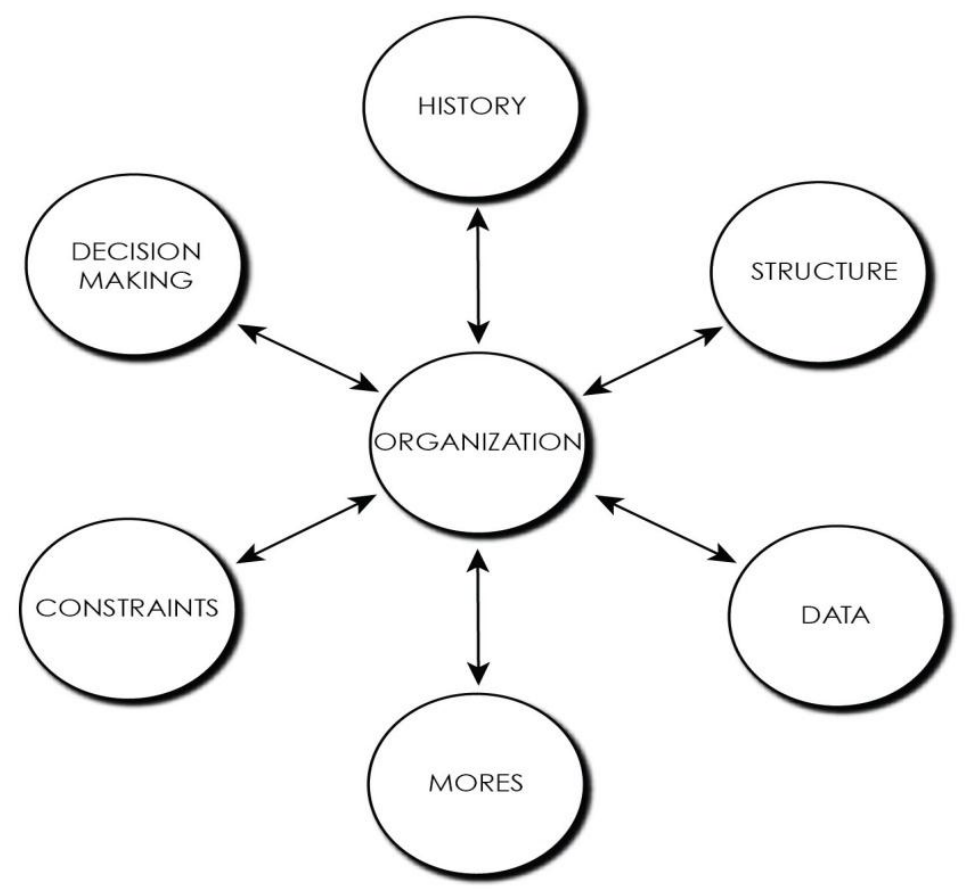

Figure 6. Organization and the environment 


\section{Where Do We Go from Here? Future Research}

This article reviewed landmark historical and current research focusing upon the failures and inadequacies associated with organizational diagnosis and development efforts including the role of the consultant in this dual process. It further proposes a psychoanalytic model to serve as an organizational behavior blueprint. The model developed and processes identified are an intensive effort to explore behavioral systems. Its goal was to describe the early stages in the growth of an organization and its human resources-its people. The model synthesized the research of psychoanalytic theory and organizational development (OD) processes in examining the components and effects of pain, affection, aggression, dependency and ego-ideal as interrelated forces.

Examining relationships between intervention levels and component parts is the primary step to be taken in diagnosing, developing and changing the organization and environmental domain to an organic, reality-centered entity. This will be the challenge for current and future OD researchers to further examine. The intra and interrelationships between the four intervention levels (individual, group, organization and environment) and the 30 variables uniquely, presents an overwhelming task for the organizational development researcher as well as a practitioner (consultant) to diagnose, describe and hopefully change as the combination of psychoanalytic elements and organizational processes are complex and enigmatic at the individual/group levels with a multiplier effect illuminating the organizational and environment domains. This area of organizational behavior and OD has been underrepresented for the past 30 years with some key research recently appearing along the way but the major issues are still there and in need of possibly quantifying it empirically for current and future scholars. The development of a model intended to diagnose the individual and total elements of the system are the primary step to be taken in this change effort for future effectiveness and success. That will be a key challenge for those interested in psychoanalytic theory as it impacts organizational functioning.

\section{REFERENCES}

[1] Appelbaum, S. (1973). Just what should an organizational development specialist do? Personnel Journal 52, pp. 222-223.

[2] Appelbaum, S. Management by Cooperation: The Views of Seven Chief Executive Officers", University of Michigan Business Review, Vol. 29, No. 6, November 1977, pp. 19-27.

[3] Appelbaum, S. Habashy, S., Malo, J-L., and Shafiq, H.,"
(2012), Back to The Future: Revisiting Kotter's 1996 Change Model", Journal of Management Development, Vol. 31, No.8, pp.764.782.

[4] Argyris, C. (1960). Individual actualization in complex organizations. Mental Health, 44, pp. 226-237.

[5] Argyris, C. (1964). Integrating the Individual and the Organization, John Wiley and Sons, New York. pp. 27-40.

[6] Armenakis, A. and Harris, S. 2009. Reflections: our journey in organizational change research and practice. Journal of Change Management, 9(2): 127-142.

[7] Blake, R. R., \& Mouton, J. S. (1964). The managerial grid: key orientations for achieving production through people. Houston, Texas: Gulf Pub. Co.

[8] Bunjak, A., Černe, M. and Wong, S. (2019), "Leaderfollower pessimism (in) congruence and job satisfaction: The role of followers' identification with a leader", Leadership \& Organization Development Journal, Vol. 40, No. 3, pp. 381-398.

[9] Bushe, G. R. and Marshak, R. J. (2009). Revisioning organization development: diagnostic and dialogic premises and patterns of practice. Journal of Applied Behavioral Science, 45: 348-368.

[10] Furgoch, K (2016), Organizational Diagnosis: What You Need to Know and Why Organizations Need Our Help, News \& Press: HRmatters - online!

[11] French, W. (1972). Organizational development: objectives, assumptions and strategies. In N. Margulies and A. Raia (Eds.), Organizational Development: Values, Process and Technology, McGraw-Hill, New York. pp. 31-49.

[12] Halfhill, T. R., Huff, J. W., Johnson, D. A., Ballentine, R. D., \& Beyerlein, M. M. (2002). Interventions that work (and some that don't). An executive summary of the organizational change literature. In R.L. Lowman (Ed.), Handbook of organizational consulting psychology (pp. 619-644). San Francisco: Jossey-Bass.

[13] Herzberg, Frederick (1968). "One More Time: How Do You Motivate Employees?". Harvard Business Review. 46 (1): 53-62

[14] Kotter, J.P. (1996), Leading Change, Harvard Business School Press, Boston, MA

[15] Lawrence, P. and J. Lorsch (1969). Developing Organizations: Diagnosis and Action, Addison-Wesley, Reading, Mass. pp. 5-99.

[16] Levinson, H. (1970). Executive Stress, Harper and Row, New York. pp. 15-197.

[17] Levinson, H. (1972). Organizational Diagnosis, Harvard University, Cambridge, Mass. pp. 4-12.

[18] Levinson, H. (2002a). Assessing organizations. In R. L. Lowman (Ed.), Handbook of organizational consulting psychology (pp. 315-343). San Francisco: Jossey-Bass

[19] Levinson, H. (2002b). Organizational assessment: A step-by-step guide to effective consulting. Washington, DC: American Psychological Association.

[20] Levinson, H. (2002c). Psychological consultation to 
organizations: Linking assessment and intervention. In R. L. Lowman (Ed.), Handbook of organizational consulting psychology (pp. 415-449). San Francisco: Jossey-Bass.

[21] Little, J. (1970). Models and managers: the concept of a decision calculus. Management Science 16, pp. 466-485.

[22] Lippitt, G. (1973). Visualizing Change: Model Building and the Change Process, NTL Learning Resources, Fairfax, Virginia, pp. 2-346.

[23] Lowman, R. (2005), Importance of Diagnosis in Organizational Assessment: Harry Levinson's Contributions, in the The Psychologist-Manager Journal, 8, No.1, pp. 17-28.

[24] Lundberg, C. (2008). Organization development diagnosis. In The Handbook of Organization Development, Edited by: Cummings, T. 137-150. Thousand Oaks, CA: SAGE

[25] Maslow, A. H. (1943). A theory of human motivation. Psychological Review, 50(4), 370-396.

[26] McGregor, D. M. (1957). The Human Side of Enterprise.” In Adventure in Thought and Action. Proceedings of the Fifth Anniversary Convocation of the School of Industrial Management, Massachusetts Institute of Technology, Cambridge, MA, Cambridge, MA: MIT,

[27] Mulder, P. (2020). Kotter's 8 Step Change Model, Toolshero,

[28] https://www.toolshero.com/change-management/8-step-cha nge-model-kotter/

[29] Noolan, J. A.C. 2006. "Organization diagnosis phase". In The NTL Handbook of Organization Development and Change: Principles, Practices, and Perspectives, Edited by: Jones, B. and Brazzel, M. 192-211. San Francisco: Pfeiffer.

[30] NTL Learning Resources, Fairfax, Virginia. pp. 2-346.

[31] March, J. and H. Simon (1958). Organizations, John Wiley and Sons, New York. pp. 84-85.

[32] Margulies N. and J. Wallace (1973). Organizational Change: Techniques and Applications, Scott, Foresman, Glenview, Illinois. pp. 7-151.

[33] McKenney J. and P. Keen (1974). How managers minds work. Harvard Business Review 52, pp. 79-90.

[34] Parveen, M. and Adeinat, I. (2019), Transformational leadership: does it really decrease work-related stress? Leadership \& Organization Development Journal, Vol. 40, No. 8 , pp. 860-876.

[35] Pearse, R. (1974). Manager to Manager: What Managers think of Management Development, AMACOM, New York. pp. 2-4.

[36] Smith, R. R. (2019). Building trust for a positive employee experience. People Matters. 1-3. Research Collection Lee Kong Chian School of Business., Singapore Management University.

[37] Spencer, B. (1974). Maturity and organizational growth pains. Personnel Journal 59, pp. 697-709.

[38] Stern, S. D. C., Cifu, A. S. and Altkorn, D. (2010). Symptom to Diagnosis: An Evidence-Based Guide, New
York: McGraw-Hill.

[39] Todnem, R. (2005), “Organizational change management: a critical review", Journal of Change Management, Vol. 5, No. 4, pp. 369-80.

[40] Vilkinas, T., Murray, D. and Chua, S. (2019), Effective leadership: Considering the confluence of the leader's motivations, behaviours and their reflective ability, Leadership \& Organization Development Journal, Vol. 41, No. 1, pp. 147-163.

[41] Vosoughi, M. (2019), Organizational Development Consulting: A Study of Expert Consultants' Key Strategies, University of North Florida, Graduate Theses and Dissertations. 550.

[42] Wong, A., Wang, X., Wang, X. and Tjosvold, D. (2019), Ethical leaders manage conflict to develop trust, Leadership \& Organization Development Journal, Vol. 41, No. 1, pp. 133-146. 